\title{
Use of Maraviroc in the prevention and Treatment of Immune Reconstitution Inflammatory Syndrome in Natalizumab-Associated Progressive Multifocal Leukoencephalopathy
}

\author{
Deepti Anbarasan ${ }^{1 *}$, Jonathan Howard ${ }^{1,2}$ and Lana Zhovtis Ryerson ${ }^{1,2}$ \\ ${ }^{1}$ NYU School of Medicine, New York, USA \\ ${ }^{2}$ NYU Langone Medical Center, Comprehensive Multiple Sclerosis Care Center, New York, USA
}

"Corresponding author: Deepti Anbarasan, Department of Neurology,New York University School of Medicine, 240 East 38th Street - 20th Floor, New York, NY, USA 10016, Tel: 9082511607; E-mail: da446@med.nyu.edu

Received date: Jul 25, 2014, Accepted date: Sep 16, 2014, Published date: Sep 19, 2014

Copyright: (C) 2014 Anbarasan D, et al. This is an open-access article distributed under the terms of the Creative Commons Attribution License, which permits unrestricted use, distribution, and reproduction in any medium, provided the original author and source are credited.

\begin{abstract}
We discuss the case of a patient with Progressive Multifocal Leukoencephalopathy (PML), developed after treatment with Natalizumab for 42 months for relapsing remitting multiple sclerosis (RRMS). Imaging was consistent with wide-spread PML with features that portended a high risk of development of Immune Reconstitution Inflammatory Syndrome (IRIS). After completion of plasmapheresis, she was started on oral maraviroc, chemokine receptor 5 antagonists. Our patient did not develop radiological signs or symptoms of clinical IRIS and tolerated maraviroc without adverse side-effects. We propose that maraviroc merits further examination as an agent that may prevent IRIS in patients with natalizumab-associated PML.
\end{abstract}

Keywords: Immune reconstitution inflammatory syndrome; Natalizumab; Maraviroc; Progressive multifocal leukoencephalopathy

\section{Case summary}

A 49-year-old, HIV negative woman with a history of RRMS for twelve years, presented for a second opinion for complaints of three months of worsening ataxia and dysarthria. Her current symptoms had begun with numbness in the lips, which then progressed to dysarthria and dysgeusia and later to difficulties with balance and cognition. She had been known to be JC virus (JCV) antibody-positive and had been maintained on natalizumab for 42 months with the last infusion being given one month prior to presentation.

Brain magnetic resonance imaging (MRI) with and without gadolinium revealed multiple enhancing T2/FLAIR hyperintensities in the bilateral middle cerebellar peduncles and the pons in addition to a non-enhancing T2/FLAIR hyperintensity in the right parieto-occipital white matter that was associated with mild mass effect. Imaging findings were consistent with wide-spread PML (Figure 1).

Cerebrospinal fluid analysis confirmed the diagnosis with an elevated JC viral load of 390 copies/mL detected using a polymerase chain reaction assay performed by Focus Diagnostics.

The patient underwent plasmapheresis to help escalate natalizumab clearance and was started on oral maraviroc $300 \mathrm{mg}$ twice a day. The patient remained free of clinical Immune Reconstitution Inflammatory Syndrome (IRIS), although consecutive MRI brain imaging studies showed faint peripheral enhancement in some of the previously identified T2/FLAIR hyperintense lesions (Figure 1). Patient's clinical status and MRI abnormalities attenuated over 6 months of treatment and maraviroc was tapered. No adverse sideeffects related to maraviroc were observed during the course of treatment.

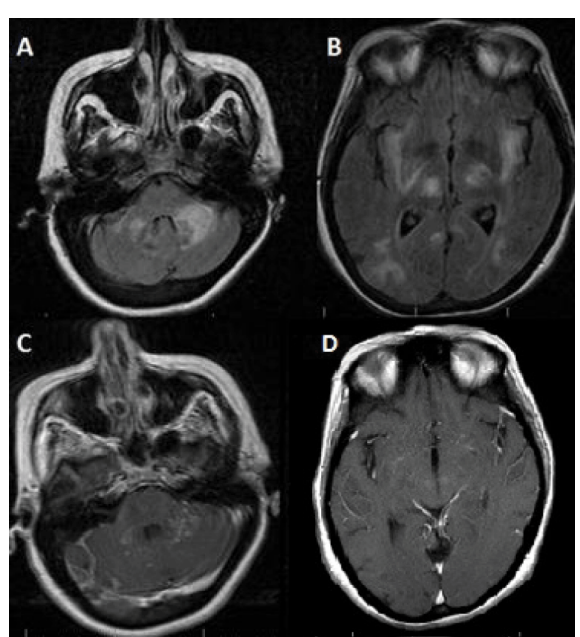

Figure 1: MRI T2-weighted/FLAIR axial images. Image IA and IB: MRI T2-weighted/FLAIR axial images show hyperintensities in the bilateral middle cerebellar peduncles, bilateral thalami, bilateral subinsular white matter, and right parieto-occipital region white matter. Image IC and ID. MRI T1-weighted axial images with gadolinium show faint peripheral enhancement in lesions around the bilateral middle cerebellar peduncles, bilateral thalami, and bilateral subinsular white matter.

At time of follow-up 12 months after initiation of treatment with maraviroc, the patient continued to exhibit mild dysarthria, facial numbness, and ataxia. MRI brain exhibited near-resolution of all T2/ FLAIR supratentorial and infratentorial hyperintensities with only minimal residual gadolinium enhancement detected. No new MS 
Citation: Anbarasan D, Howard J, Ryerson LZ (2014) Use of Maraviroc in the prevention and Treatment of Immune Reconstitution Inflammatory Syndrome in Natalizumab-Associated Progressive Multifocal Leukoencephalopathy. J Mult Scler 1: 116. doi:10.4172/jmso.1000116

Page 2 of 2

disease activity was noted. She was started on subcutaneous glatiramer acetate injections $20 \mathrm{mg}$ daily for ongoing treatment of MS.

\section{Discussion}

This case study illustrates the potential utility of maraviroc in the prevention of clinical symptoms of IRIS, an entity that is considered to be an almost universal complication that occurs with or without plasmapheresis in patients with natalizumab-associated PML (NTZPML) [1]. IRIS is a major cause of mortality and morbidity in patients with NTZ-PML and is characterized either by sudden clinical deterioration or by gadolinium enhancement and/or mass effect on brain MRI [2]. Its pathophysiology is linked to the rapid trafficking of JCV-specific T cells into the central nervous system, which aids in the destruction of infected oligodendrocytes but also results in collateral damage [3]. Although glucocorticoids are the treatment of choice for IRIS, recent evidence suggests that they may limit JC viral clearance and may not be effective for the prevention of IRIS in NTZ-PML. As such, new therapeutic modalities need to be evaluated for the management of IRIS in these patients.

Maraviroc is approved for HIV-treatment as part of combination antiretroviral therapy and is an allosteric inhibitor of chemokine receptor 5-positive (CCR5+) T cells [4]. CCR5+ blockade results in the inhibition of cellular trafficking and activation of effector $\mathrm{T}$ cells to sites of infection or inflammation. Since IRIS is associated with an increase in pathogen-specific inflammatory responses including upregulation of CCR5+ immune T-cells, maraviroc has been suggested to have utility in the prevention of IRIS associated with antiretroviral initiation in patients with HIV [5]. This led to the consideration that maraviroc may be used in the prevention of IRIS in patients with NTZ-PML, but no clinical trials have yet been performed to evaluate this hypothesis.

One prior case study examines the use of maraviroc in the management of IRIS associated with NTZ-PML. Giacomini et al presented the case of a patient with MS who presented with NTZ-PML and was treated with maraviroc [6]. They demonstrated decreased proportions of CCR5+ immune cells in the cerebrospinal fluid, indicating that maraviroc selectively limits cell trafficking into the central nervous system. They posited that the drug may have contributed to the initial prevention and to active treatment of IRIS in this case.

Our patient presented with three months of progressive clinical deterioration that was consistent with PML in the context of natalizumab use and JCV seropositivity. Her initial brain MRI was consistent with both PML as well as early IRIS, given the presence of both gadolinium enhancement and mass effect even prior to the removal of natalizumab with plasmapheresis [1]. Such patients with early immunologic rebound in NTZ-PML typically have earlier and more severe clinical symptoms of IRIS in addition to worse survival rates compared to those patients who develop imaging changes only after natalizumab removal. Nonetheless, our patient did not develop clinical symptoms of IRIS after undergoing plasmapheresis and starting treatment with maraviroc. She showed slow improvement of her neurological deficits and imaging findings over the course of nine months.

Our experience, in addition to that of Giacomini et al, demonstrates that even patients who are considered to be at high risk for developing clinical symptoms of IRIS may avoid this complication [6]. We propose that this may be attributable to the effects of maraviroc. Safety and tolerability studies to date have demonstrated that this drug has minimal interactions with major drug-metabolizing enzymes and is well tolerated at clinically relevant doses without serious adverse effects $[7,8]$. Given the favorable safety profile of maraviroc and the potentially devastating consequences of IRIS, we advocate for a clinical trial of this medication in natalizumab-associated PML.

\section{Acknowledgements}

This research received no specific grant from any funding agency in the public, commercial, or not-for-profit sectors.

\section{References}

1. Tan IL, McArthur JC, Clifford DB, Major EO, Nath A (2011) Immune reconstitution inflammatory syndrome in natalizumab-associated PML. Neurology 77: 1061-1067.

2. McCarthy M, Nath A (2010) Neurologic consequences of the immune reconstitution inflammatory syndrome (IRIS). Curr Neurol Neurosci Rep 10: 467-475.

3. Antoniol C, Jilek S, Schluep M, Mercier N, Canales M, et al. (2012) Impairment of JCV-specific T-cell response by corticotherapy: effect on PML-IRIS management? Neurology 79: 2258-2264.

4. Parra J, Portilla J, Pulido F, Sánchez-de la Rosa R, Alonso-Villaverde C, et al. (2011) Clinical utility of maraviroc. Clin Drug Investig 31: 527-542.

5. Boesecke C, Pett SL (2012) Clinical studies with chemokine receptor-5 (CCR5)-inhibitors. Curr Opin HIV AIDS 7: 456-462.

6. Giacomini PS, Rozenberg A, Metz I, Araujo D, Arbour N, et al. (2014) Maraviroc and JC virus-associated immune reconstitution inflammatory syndrome. N Engl J Med 370: 486-488.

7. Gulick RM, Fatkenheuer G, Burnside R, Hardy WD, Nelson MR, et al. (2014) Five-year safety evaluation of maraviroc in HIV-1-infected treatment-experienced patients. J Acquir Immune Defic Syndr 65: 78-81.

8. Abel S, van der Ryst E, Rosario MC, Ridgway CE, Medhurst CG, et al. (2008) Assessment of the pharmacokinetics, safety and tolerability of maraviroc, a novel CCR5 antagonist, in healthy volunteers. Br J Clin Pharmacol 65 Suppl 1: 5-18. 